\title{
Assessing the format of the presentation of text in developing a Reading Strategy Assessment Tool (R-SAT)
}

\author{
Sara Gilliam, Joseph P. Magliano, and Keith K. Millis \\ Northern Illinois University, DeKalb, Illinois \\ AND \\ IRWIN LEVINSTEIN AND ChUTIMA BOONTHUM \\ Old Dominion University, Norfolk, Virginia
}

\begin{abstract}
We are constructing a new computerized test of reading comprehension called the Reading Strategy Assessment Tool (R-SAT). R-SAT elicits and analyzes verbal protocols that readers generate in response to questions as they read texts. We examined whether the amount of information available to the reader when reading and answering questions influenced the extent to which R-SAT accounts for comprehension. We found that R-SAT was most predictive of comprehension when the readers did not have access to the text as they answered questions.
\end{abstract}

Skilled readers are purposeful and active, employing reading strategies that support learning as they read a text (Pressley \& Afflerback, 1995; Snow, 2002). Skilled readers use experience, knowledge of the world, vocabulary, and reading strategies to make sense of a text and to maximize the information and meaning they extract from it. They are able to monitor their level of understanding while reading and to adjust their reading strategies when understanding is lost (McNamara, Levinstein, \& Boonthum, 2004). Young, less skilled readers, in contrast, exhibit a lack of such ability (Cordón \& Day, 1996; Magliano \& Millis, 2003). In order to help less skilled readers become better readers, it is necessary to be able to teach them these strategies and to measure whether they use them spontaneously. Unfortunately, current tests of reading skill are not designed to evaluate whether readers employ reading strategies.

We are constructing a new test of reading comprehension called the Reading Strategy Assessment Tool (R-SAT) designed to evaluate how readers employ reading strategies. R-SAT extends a growing movement in test development that has students write open-ended responses rather than answer multiple-choice questions (Magliano \& Millis, 2003). R-SAT elicits and analyzes verbal protocols that readers generate as they read narrative, historical, and scientific texts. After reading preselected target sentences, the R-SAT reader is told, "Report your thoughts regarding your understanding of the sentence in the context of the passage" (the indirect method) or asked a wh- question that probes the reader's textbase and situation model (the direct method). The indirect method is designed to produce verbal protocols that reveal the use of various reading strategies. The direct method is designed to as- sess the comprehension of the text by requiring a reader to generate answers to questions that are found primarily in the prior discourse.

In order to evaluate which reading strategies a reader is using, the reader must report his or her mental representation of the text. The strength of a reader's memorial representation of a text may depend on how much information is available to the reader at the time he or she is prompted with a question. Some researchers have presented one sentence at a time with no text available at the time of the question prompt (Trabasso \& Magliano, 1996a, 1996b). Other researchers have made the whole text available at the time of the question prompt (Chi, Bassok, Lewis, Reimann, \& Glaser, 1989; Muñoz, Magliano, Sheridan, \& McNamara, 2006). Both of these text presentation formats have produced protocols which have been found indicative of comprehension. However, there has not been a systematic study that has examined the impact of the available text on the reading strategies revealed in verbal protocols and the extent to which these strategies are indicative of comprehension. The amount of text available when readers receive the indirect and direct questions in the context of R-SAT may have implications for the quality of student answers to these questions. In the next section, we discuss the reading strategies assessed by R-SAT that may be influenced by the amount of content available when verbal protocols are produced.

\section{R-SAT AND READING STRATEGIES}

Think-aloud methodologies have been extensively used to assess and examine comprehension processes (Coté \&

S. Gilliam, sgilliam@niu.edu 
Table 1

Text Excerpt From the Passage "Understanding Life"

\begin{tabular}{clr}
\hline $\begin{array}{c}\text { Sentence } \\
\text { Number }\end{array}$ & \multicolumn{1}{c}{ Sentence } & \multicolumn{1}{c}{ Type } \\
\hline 1 & $\begin{array}{l}\text { Throughout history, humans have sought to understand } \\
\text { how life arises. } \\
\text { Curiously, at one time, many people thought that many } \\
\text { animals were spawned from dead or nonliving matter. }\end{array}$ & Distal sentence \\
2 & $\begin{array}{l}\text { This theory can be traced back to the ancient Greeks. } \\
\text { It's called spontaneous generation. }\end{array}$ & $\begin{array}{l}\text { Local sentence } \\
\text { Current sentence }\end{array}$ \\
4 & $\begin{array}{l}\text { Report your thoughts regarding your understanding of } \\
\text { the sentence in the context of the passage. }\end{array}$ & $\begin{array}{l}\text { Indirect question prompt } \\
5\end{array}$ \\
\hline
\end{tabular}

Goldman, 1999; Ericsson \& Simon, 1993; Pressley \& Afflerbach, 1995; Trabasso \& Magliano, 1996b; Whitney \& Budd, 1996; Zwaan \& Brown, 1996). R-SAT is based on research demonstrating that strategies when thinking aloud are indicative of comprehension (Magliano \& Millis, 2003; Millis, Magliano, \& Todaro, 2006). For example, Magliano and Millis had people read text and think aloud, either after every sentence or after preselected sentences. Using Latent Semantic Analysis (LSA) to measure the semantic overlap between the current sentence and individual protocols, Magliano and Millis found that the greater the semantic overlap, the less accurately readers performed on reading comprehension tests; the greater the overlap between the prior discourse and a reader's protocols, the better they performed on comprehension tests. In their study, Magliano and Millis made available at the time of the prompt only one sentence which required readers to elicit verbal protocols. In a separate study, Millis et al. (2006) presented the entire text at the time of the prompt. The text presentation methods used in both studies were indicative of comprehension. As mentioned above, there has not been a study examining the impact of the amount of text displayed at the time of the prompt on the use of reading strategies and the extent to which such strategies are correlated with outcome measures of comprehension.

R-SAT requires readers to comprehend short texts from three different genres: science, history, and narrative. As mentioned earlier, readers answer direct or indirect questions after particular target sentences. A word matching algorithm is employed to establish which strategy or strategies a reader may be using. The word matching algorithm is set up to count the number of words in common between a protocol and a particular reference source thought to be indicative of reading strategy. Think-aloud protocols can reveal content from at least three sources: the current sentence, prior text, and/or world knowledge (Todaro, Magliano, Millis, Kurby, \& McNamara, 2006; Trabasso \& Magliano, 1996a). This content reflects the inferences and reading strategies produced by a reader at a given sentence. For example, a reader who produces information from a sentence just read is probably producing a paraphrase of that sentence. Paraphrasing occurs when a reader rephrases all or part of the current sentence. Consider the example text excerpt from "Understanding Life" in Table 1 and the example verbal protocols for the same text in Table 2, which were produced at Sentence 5 of the text. Clause 3 for Reader 2 is a paraphrase because the participant explicitly states ideas from the current sentence. Paraphrasing allows readers the opportunity to discuss the current discourse content in terms that are more familiar to them (McNamara et al. 2004). This may help readers to construct a meaningful mental representation of the text.

Bridging occurs when readers explain a sentence in terms of relevant prior text information. Bridges can be to the local discourse context if they connect the current sentence with the immediately prior sentence, which should still be available in working memory, or to the distal discourse context, requiring readers to activate that knowledge from episodic memory (Trabasso \& Magliano, 1996a). For example, Clauses 1 and 2 for Reader 1 reflect local bridging, because the participant mentions events described in the immediately preceding sentence (see the text in Table 1). In contrast, Clause 3 for Reader 3 reflects distal bridging, because it mentions information contained in Sentence 2. Bridging allows readers to create local and global coherence, which is necessary for overall comprehension.

Elaborations occur when the reader elaborates or explains the text in terms of relevant world knowledge. For example, Clause 3 for Reader 3 is considered an elaboration because the reader mentions a topic not discussed in the text - reincarnation - that may serve as an explanation for the event - spontaneous generation - that is discussed in the text.

The goal of this study was to evaluate three different text-presentation formats, in the context of R-SAT, in which there is a varying amount of text available to the

Table 2

Examples of Reading Strategies Elicited by Verbal Protocols for the Text "Undersanding Life"

\begin{tabular}{|c|c|c|c|}
\hline Reader & Clause & Protocol & Strategy \\
\hline 1 & $\begin{array}{l}1 \\
2 \\
3\end{array}$ & $\begin{array}{l}\text { The Greeks first thought } \\
\text { of life tracing back } \\
\text { to dead matter }\end{array}$ & $\begin{array}{l}\text { Local bridge } \\
\text { Local bridge } \\
\text { Distal bridge }\end{array}$ \\
\hline 2 & $\begin{array}{l}1 \\
2 \\
3\end{array}$ & $\begin{array}{l}\text { Spontaneous generation } \\
\text { Is that people think life } \\
\text { Spontaneously generates }\end{array}$ & $\begin{array}{l}\text { Paraphrase } \\
\text { Distal bridge } \\
\text { Paraphrase }\end{array}$ \\
\hline 3 & $\begin{array}{l}1 \\
2 \\
3\end{array}$ & $\begin{array}{l}\text { Greeks believed } \\
\text { In spirits and living forever } \\
\ldots \text { through reincarnation. }\end{array}$ & $\begin{array}{l}\text { Local bridge } \\
\text { Elaboration } \\
\text { Elaboration }\end{array}$ \\
\hline
\end{tabular}


reader at the time of the question prompt. We are particularly interested in the degree of impact on the indirect question prompts, because they reflect the reading strategies. However, the direct questions are also of interest, because they provide information about how readers, in their mental search for an answer, use the text that is present. In a single-sentence absent condition, currently employed by R-SAT, the text was presented to the student one sentence at a time and no text was available when the direct and indirect questions appeared. This format required the reader to access their mental representation of the text from memory in order to answer a question about the text. In a single-sentence present condition, the text was presented one sentence at a time, but the target sentence remained on the screen at the question prompt. In this condition, the sentence that was available at the time of the prompt may have provided a retrieval cue to access the prior discourse from memory. In a whole-text condition, the text was presented one sentence at a time in a cumulative fashion so that all prior text, up to and including the target sentence, was available to the reader when the reader answered the questions. In the whole text condition, readers may have been able to use the text to reconstruct the information portrayed in the text. Generally, the less text available at the prompt site, the fewer resources available for working memory processes.

\section{METHOD}

\section{Participants}

One hundred ninety-eight undergraduates from Northern Illinois University participated in this study for credit in an introductory psychology course (67 participated in the whole text, 66 participated in the single-sentence present condition, 65 in the single-sentence absent condition).

\section{Materials}

Participants' comprehension skill was measured on the basis of their performance on short-answer (SA) questions associated with two texts, one a historical narrative, the other a science text, both comparable in length and difficulty to the R-SAT texts. Ten SA questions were constructed for each text. Five of these questions were text based, and five were situation model questions. Ideal answers were constructed for each of the SA questions. The ideal answers were decomposed into necessary idea units. Each essay was scored on a 4-point scale, as follows: 0 , incorrect; 1 ; vague but correct; 2 , partially complete; and 3, complete. Interrater reliability for scoring these answers was high $(r=.92)$. A total score for each participant was calculated by creating a percentage from the total points earned on the short answer divided by the total points possible.

\section{The Mechanics of R-SAT}

R-SAT is administered on personal computers in a Web-based environment. The tool was developed in the Java Language, using the Apache Tomcat Web server and a MySQL database. The texts are presented in a black font on a gray field, left justified near the top of the computer screen. The title of each text remains centered at the top of the screen while participants read the entire text. In the current version of R-SAT adopted from the approach taken by Magliano and Millis (2003), only one sentence of a text is shown on the screen, but that sentence is removed when the reader receives the question prompts (Magliano, Millis, Gilliam, Levinstein, \& Boonthum, 2006). Participants navigate forward through the text by clicking on a "next" button located near the bottom left of the computer screen. New paragraph markers appear when there is a shift to a new paragraph. After participants click the "next" button, one of three things can occur: For a nontarget sentence, the next sentence will appear; for a target sentence, a response box appears to the right of the "next" button with the prompt "Report your thoughts regarding your understanding of the sentence in the context of the passage" above the box; for direct questions, the target sentence is removed from the screen when the question and the response box appear. Participants type their answers in the response box. They click the "next" button when they are finished, after which the response box disappears and the next sentence is presented. Responses are recorded on a computer server. The texts are presented in random order to the participants.

The R-SAT protocol evaluation program was developed in Java. As briefly described above, it uses word matching algorithms to evaluate the protocols. Words, excluding stop-words - commonly used words such as articles and prepositions - are compared with a number of information sources: the current sentence (words in the target sentence); the local prior sentence (words in the preceding sentence); distal prior sentences (words in all prior sentences, excluding the immediately preceding one); later sentences (words in later sentences); and an ideal answer (words in the ideal answer of a given target sentence). This is accomplished in two ways: by literal word matching and by Soundex matching.

In literal word matching, words are compared character by character. A match of the first $75 \%$ of the characters in a word in the target sentence is called a literal match. This also includes removing the suffixes $-s,-d,-e d$, -ing, and -ion from the end of each word. For example, if the trainee's self-explanation contains the word thunderstorm (even with a misspelling), it still counts as a literal match with words in the target sentence, because the first nine characters are exactly the same. On the other hand, if the self-explanation contains the word thunder only, it will not get a match with the target sentence.

The Soundex matching algorithm compensates for misspellings by mapping similar characters to the same Soundex symbol (Christian, 1998). Words are transformed to their Soundex code by retaining the first character, dropping the vowels, and converting other characters into Soundex symbols. If the same symbol occurs more than once consecutively, only one occurrence is retained. For example, thunderstorm will be transformed to $t 8693698$; communication to $c 8368$. Note that the latter example was originally transformed to $c 888368$ and two 8 s were dropped ( $m$ and $n$ are both mapped to 8 ). If the trainee's self-explanation contained thonderstorm or tonderstorm, both would be matched with thunderstorm; this is called a Soundex match. An exact Soundex match is required for short words (i.e., those with fewer than 6 alpha characters), due to the high number of false alarms when Soundex is used. For longer words, a match on the first 4 Soundex symbols suffices.

For indirect questions, each word in the student's protocol is checked in the following order: (1) whether it appears in the current sentence; (2) whether it appears in the local sentence; (3) whether it appears in the distal sentence; and (4) whether it appears in a later sentence. Each check includes both literal word and Soundex matching. Also, there are two approaches in matching: inclusive or exclusive. The inclusive approach checks all information sources, whereas the exclusive approach stops with the first match; that is, if a word is matched under the current sentence, it will not be checked for local, distal, and later sentences. The exclusive approach has the effect of eliminating overlap between information sources by assuming that a word that appears in two information sources refers to the one closest to the current sentence. This approach was taken because we assumed that content words in the protocols that were based on the current and prior discourse came from one of the most immediate discourse contexts to working memory. This assumption was adopted with prior research using LSA to analyze the protocols (Magliano, Millis, Levinstein, \& Boonthum, 2005; Magliano, Wiemer-Hastings, Millis, Muñoz, \& McNamara, 2002; Millis, Kim, Todaro, Magliano, Wiemer-Hastings, \& McNamara, 2004). Content words (i.e., those not in the stop-word list) that are not matched with 
these 4 information sources are tagged as not mentioned words. A new words variable was created by summing not-mentioned and later words. For the direct questions, each word in the student's answer is checked against the ideal answer, using both literal word and Soundex matching.

Finally, participants were administered R-SAT on personal computers in a web-based environment. Instructions, text materials, and verbal protocols were all administered on the computer. R-SAT includes 6 texts from three different genres: 2 science, 2 historical narratives, and 2 fictive narratives. Three versions of R-SAT were developed for this experiment on the basis of the amount of available text. In the first and second versions, text was displayed one sentence at a time on a computer screen. In the third version, text was displayed in a cumulative fashion, a sentence at a time, so that the entire text up to that point in time was displayed. Title and paragraph information was provided to all readers while they read. Each target sentence was followed by a question prompt. In the single-sentence absent version of R-SAT, no text was available to the reader at the time of the prompt. In the single-sentence present version of R-SAT, the current sentence was available at the time of the prompt, and in the whole-text version of R-SAT, all of the text up to the time of the prompt was displayed at the time of the prompt.

Target sentences for the question prompts were chosen on the basis of two criteria: the theoretical possibility that readers could bridge the current sentence to previous ones, and the data from a previous study indicating that protocols produced at the target sentences were correlated with outcome measures of comprehension (Magliano et al., 2005). As previously mentioned, there were two methods for questioning at the prompt sites: a direct method and an indirect one. The majority of the direct questions consisted of whquestions, and in particular why questions (e.g., Why does a tumor develop?). Ideal answers generated by the second and third authors were used to evaluate the quality and richness of the participants' answers. The prompt for the indirect questions was "Report your thoughts regarding your understanding of the text so far."

\section{Procedure}

The experiment occurred in two sessions. Session 1 occurred in groups of about 30 students and Session 2 in groups of about 6 students, a week or so later. In Session 1, participants received a comprehension test packet containing the two comprehension texts and corresponding SA questions. Each text fit on a single piece of paper. After each text reading, the participants were given arithmetic problems to displace the contents of working memory. After completing these problems, the participants were presented the $10 \mathrm{SA}$ questions. There were five questions per sheet of paper. The texts were not available when the participants answered the SA questions. Participants were allowed as much time as needed to complete this phase of the experiment.

Session 2 occurred in a computer laboratory that could accommodate up to six participants at a time. After being given an instructionand-practice packet, participants were randomly assigned one of three versions of R-SAT. The experimenters read through the instructions with the participants, who were told that they would be reading a series of short texts that would appear, one sentence at a time, on the computer screen. Participants were also informed that, while reading, they would periodically be asked two types of questions: When shown the prompt "What are you thinking now," they were to report whatever thoughts came to mind about what they had just read, and to focus on how they understood the sentence just read in terms of the larger text. They were to avoid vague or uninformative responses, such as "OK, I knew this," or "I didn't know this." Participants were told that some of the direct questions would require very specific answers, and that they were to answer those questions as completely as possible. They received a practice packet consisting of a paper-and-pencil example mirroring the method used for the RSAT computer-based tool. A short five-sentence text was used. Each sentence was presented on a separate sheet of paper. After the first two sentences participants were asked an indirect question, and after the third sentence they were asked a direct question. They answered these questions on paper and the experimenter read through the answers to make sure that they had understood the instructions. If the answers to the indirect questions were vague or uninformative, the experimenter was instructed to remind participants that they were to report their thoughts regarding their understanding of the text, which meant that they were to discuss the content of their understanding. The experimenters were careful not to provide information, such as prior texts or relevant world knowledge, regarding the nature of that content. After completing the practice, participants began using the computer-based R-SAT tool. This phase of the experiment took approximately an hour to complete.

\section{R-SAT Scoring of the Protocols}

The direct and indirect protocols were scored using the word matching algorithms described above. With respect to the direct protocols, R-SAT recorded the number of content words from the ideal answers in the protocols. There were four word counts calculated for the indirect protocols: current sentence, local sentence, distal sentences, and new words (i.e., content words not present in the current or prior sentences). These word counts corresponded to the strategies of paraphrasing, local bridging, distal bridging and elaborations, respectively.

\section{RESULTS}

There were two sets of analyses. The first set tested whether text format influenced the number of words in the different categories corresponding to the reading strategies. A second set tested whether the word counts in the three text presentation formats differentially predicted performance on the short answer questions.

\section{Reading Strategies}

Table 3 presents the means for the word counts associated with the indirect protocols as a function of the text format. A 3 (text presentation format) $\times 4$ (reading strategy) mixed ANOVA was performed to assess differ-

Table 3

Means and Standard Deviations for the Word Counts for the Current Sentence, Local Sentence, Distal Sentence, and New Words As a Function of Text Format

\begin{tabular}{|c|c|c|c|c|c|c|}
\hline \multirow[b]{3}{*}{ Predictor Variable } & \multicolumn{6}{|c|}{ Text Presentation Format } \\
\hline & \multicolumn{2}{|c|}{$\begin{array}{c}\text { Single Sentence } \\
\text { Absent }\end{array}$} & \multicolumn{2}{|c|}{$\begin{array}{c}\text { Single Sentence } \\
\text { Present }\end{array}$} & \multicolumn{2}{|c|}{$\begin{array}{l}\text { Whole } \\
\text { Text }\end{array}$} \\
\hline & $M$ & $S D$ & $M$ & $S D$ & $M$ & $S D$ \\
\hline Current sentence & 0.86 & 0.40 & 0.95 & 0.20 & 0.84 & 0.43 \\
\hline Local sentence & 0.24 & 0.24 & 0.22 & 0.48 & 0.32 & 0.22 \\
\hline Distal sentence & 1.02 & 0.52 & 0.95 & 0.22 & 0.92 & 0.51 \\
\hline New words & 3.91 & 1.60 & 3.53 & 1.77 & 3.36 & 1.67 \\
\hline
\end{tabular}


ence on the average word counts. Text format was the between-participants variable and reading strategy was the within-participants variable. A main effect of strategy $\left[F(3,585)=617.98, M S_{\mathrm{e}}=0.705, p>.01\right]$ was found. Tukey's post hoc tests revealed more new words $(M=$ 3.60) were produced than words from the current $(M=$ $0.89)$ and distal $(M=0.96)$ sentences. Additionally, there were more words produced from the current and distal sentences than from the local sentences $(M=0.26)$. A format $\times$ strategy interaction approached significance $\left[F(6,585)=2.01, M S_{\mathrm{e}}=0.705, p<.06\right]$. The trend suggests that participants produced fewer new words when text was present at the prompt than when there was no text present.

A one-way ANOVA was conducted on the mean number of words produced from the ideal answers for the direct questions, which was significant $[F(2,195)=12.175$, $\left.M S_{\mathrm{e}}=0.482, p<.01\right]$. Tukey's post hoc tests revealed that more words were produced from the ideal answers in the whole-text condition $(M=2.20, S D=.72)$ than either the single-sentence absent $(M=1.64, S D=.67)$ or single-sentence present $(M=1.72, S D=.69)$ conditions, which did not differ.

\section{Predicting SA Performance}

Multiple regression analyses were used to assess the extent to which the R-SAT variables accounted for performance on the SA questions. Three separate regression analyses were conducted for each of the three format conditions. The criterion variable for each analysis was the average performance on the SA questions. The predictor variables were average word counts for the current sentence, local sentence, distal sentences, new words, and ideal answers which were force entered into the regression equations. Table 4 contains the standardized beta weights and the amount of variance explained for each of the regression analyses. All three regression equations accounted for a significant amount of the variance in SA performance, but the single-sentence conditions accounted for the most. The single-sentence absent condition accounted for slightly more variance than the single-sentence present condition. It should be noted that the whole-text condition accounted for substantially less variance than did the other two conditions. New words and the ideal answer were significant predictors of SA performance when the text was present at the time of the

Table 4

Accounting for Comprehension Across Text Presentation Formats

\begin{tabular}{lccc}
\hline & \multicolumn{3}{c}{ Text Presentation Format } \\
\cline { 2 - 4 } Predictor Variable & $\begin{array}{c}\text { Single Sentence } \\
\text { Absent }\end{array}$ & $\begin{array}{c}\text { Single Sentence } \\
\text { Present }\end{array}$ & $\begin{array}{c}\text { Whole } \\
\text { Text }\end{array}$ \\
\hline Current sentence & $-.42^{* *}$ & -.21 & -.22 \\
Local sentence & .07 & -.09 & .03 \\
Distal sentence & .12 & -.03 & -.03 \\
New words & .15 & $.036^{* *}$ & $.33^{*}$ \\
Ideal answer & $.63^{* *}$ & $.45^{* *}$ & $.23^{*}$ \\
Variance explained & $39 \%^{* *}$ & $35 \% 0^{* *}$ & $23 \%^{* *}$ \\
\hline${ }^{*} p<.05 .{ }^{* *} p<.01$. & & &
\end{tabular}

prompt. The current sentence and the ideal answer were significant predictors of SA performance when no text was available at the time of the prompt (single-sentence absent condition).

\section{DISCUSSION}

The goal of this study was to assess differences in performance on R-SAT as a function of different text formats. Although the different formats used in this study have been employed in the literature (e.g., Magliano \& Millis, 2003; Millis et al., 2006), direct comparisons among them have never been conducted. The primary difference between the single-sentence formats and the whole-text format in terms of performance on the direct and indirect questions was that participants produced more words for the ideal answers for the direct questions when the entire text was present. Presumably this occurred because readers could formulate their answers by rereading the text. In the other two conditions, participants had to generate answers based on the memorial representation for the text. Apparently, having the retrieval cue of the sentence present at the question prompt did not lead to improvements in performance on the direct questions.

More critically for the development of R-SAT, we found that having only a single-sentence format available at the time of the prompt resulted in the best measure of comprehension. Moreover, the whole-text condition led to substantially less variance accounted for than in the singlesentence conditions. Upon examining performance on the direct and indirect questions, this drop in predictive power may be the result of participants being able to answer the direct questions more completely in the whole-text condition. Also, the single-sentence version may place more of a burden on working memory resources, which are indicative of individual differences in reading comprehension skill (e.g., Just \& Carpenter, 1992).

One promise of R-SAT is that it will provide information on reading strategies used by the reader, as reflected by their indirect protocols. In the past, we have found that the more readers mention information from the current sentence, the poorer they perform on outcome measures of comprehension, but the more they produce from the prior discourse context, the better they perform (Magliano \& Millis, 2003; Millis et al., 2006; Millis, Magliano, Wiemer-Hastings, Todaro, \& McNamara, in press). Overlap with the prior discourse context should reflect the extent to which readers are establishing coherence in understanding; this is why we typically find positive correlations with comprehension measures. Although we have found these trends with R-SAT (Magliano et al., 2006), we did not find them in the current study. However, one interesting finding was that the number of new words was a significant predictor when any text was present, although they decreased in number in these conditions. It may be the case that readers rely less on world knowledge when the text is present, which may lead to this variable being a significant predictor. It appears that skilled readers are more likely to rely on appropriate reading strategies regardless of the amount of text content present. 
In summary, a text format making one sentence available at a time is a better predictor of comprehension than having the entire text present. This study confirms that the current version of R-SAT, which adopts the singlesentence absent format, is the optimal approach for developing an assessment tool of reading skill.

\section{AUTHOR NOTE}

The research was supported by Institute for Education Sciences (IES) Grant R305G040055. Any opinions, findings, and conclusions or recommendations expressed in this article are those of the authors and do not necessarily reflect the views of the IES. We thank the following individuals for all of their hard work: Mary Hecht, P.J. Perry, Stacey Todaro, Christopher Kurby, Brent Muñoz, Jim Woehrle, Danielle Mull, Siva Kancherla, Srikanth Dandotkar, and Pratap Verbenelli. Correspondence concerning this article should be addressed to S. Gilliam, Department of Psychology, Northern Illinois University, DeKalb, IL 60115 (e-mail: sgilliam@niu.edu).

\section{REFERENCES}

Chi, M. T. H., Bassok, M., Lewis, M. W., Reimann, R., \& Glaser, R. (1989). Self-explanation: How students study and use examples in learning to solve problems. Cognitive Science, 13, 145-182.

Christian, P. (1998, March). Soundex - can it be improved? Computers in Genealogy, 6(5).

CoRdón, L. A., \& DAY, J. D. (1996). Strategy use on standardized reading comprehension tests. Journal of Educational Psychology, 88, 288-295.

CotÉ, N., \& Goldman, S. R. (1999). Building representations of informational text: Evidence from children's think-aloud protocols. In H. van Oostendorp \& S. R. Goldman (Eds.), The construction of mental representations during reading (pp. 169-193). Mahwah, NJ: Erlbaum.

Ericsson, K. A., \& Simon, H. A. (1993). Protocol analysis: Verbal reports as data. Cambridge, MA: MIT Press.

Just, M. A., \& CARPENTER, P. A. (1992). A capacity theory of comprehension: Individual differences in working memory. Psychological Review, 99, 122-149.

Magliano, J. P., \& Millis, K. K. (2003). Assessing reading skill with a think-aloud procedure and latent semantic analysis. Cognition \& Instruction, 21, 251-284.

Magliano, J. P., Millis, K. K., Gilliam, S., Levinstein, I., \& BoonThum, C. (2006). Assessing the Reading Strategy Assessment Tool. Paper presented at the 47th Annual Meeting of the Psychonomic Society, Houston, TX.

Magliano, J. P., Millis, K. K., Levinstein, I., \& Boonthum, C. (2005). Developing the Reading Strategy Assessment Tool (R-SAT).
Presented at the 35th Annual Meeting of the Society for Computers in Psychology, Toronto, Ontario, Canada.

Magliano, J. P., Wiemer-Hastings, K., Millis, K. K., Muñoz, B. D., \& McNamara, D. S. (2002). Using latent semantic analysis to assess reader strategies. Behavior Research Methods, Instruments, \& Computers, 34, 181-188.

McNamara, D. S., Levinstein, I. B., \& Boonthum, C. (2004). iSTART: Interactive strategy training for active reading and thinking. Behavior Research Methods, Instruments, \& Computers, 36, 222-233.

Millis, K. K., Kim, H. J., Todaro, S., Magliano, J. P., WiemerHastings, K., \& MCNamara, D. S. (2004). Identifying reading strategies using latent semantic analysis: Comparing semantic benchmarks. Behavior Research Methods, Instruments, \& Computers, 36, 213-231.

Millis, K. K., Magliano, J. P., \& Todaro, S. (2006). Measuring discourse-level processes with verbal protocols and latent semantic analysis. Scientific Studies of Reading, 10, 251-283.

Millis, K. [K.], Magliano, J [P.]., Wiemer-Hastings, K., Todaro, S., \& MCNAMARA, D. S. (in press). Assessing and improving comprehension with Latent Semantic Analysis. In T. Landaulet, D. S. McNamara, S. Dennis, \& W. Kintsch (Eds.), Handbook of Latent Semantic Analysis. Mahwah, NJ: Erlbaum.

Muñoz, B., Magliano, J. P., Sheridan, R., \& McNamara, D. S. (2006). Typing versus thinking aloud when reading: Implications for computer-based assessment and training tools. Behavior Research Methods, 38, 211-217.

Pressley, M., \& AfFlerbach, P. (1995). Verbal protocols of reading: The nature of constructively responsive reading. Hillsdale, NJ: Erlbaum.

SNOw, C. E. (2002). Reading for understanding: Toward a research and development program in reading comprehension. Pittsburgh: Rand.

Todaro, S., Magliano, J. P., Millis, K. K., Kurby, C. A., \& McNaMARA, D. S. (2006). Understanding factors that influence the content and form of verbal protocols: The roles of the reader and the text. Manuscript submitted for publication.

Trabasso, T., \& Magliano, J. P. (1996a). Conscious understanding during comprehension. Discourse Processes, 21, 255-287.

Trabasso, T., \& Magliano, J. P. (1996b). How do children understand what they read and what can we do to help them? In M. Graves, P. van den Broek, \& B. Taylor (Eds.), The first R: A right of all children (pp. 158-181). New York: Teachers College, Columbia University Press.

Whitney, P., \& Budd, D. (1996). Think-aloud protocols and the study of comprehension. Discourse Processes, 21, 341-351.

ZwaAn, R. A., \& Brown, C. M. (1996). The influence of language proficiency and comprehension skill on situation-model construction. Discourse Processes, 21, 289-328.

(Manuscript received December 8, 2006; revision accepted for publication March 13, 2007.) 